DOI:10.30842/ielcp230690152317

E. V. Grigoryeva

(Берлин, Германия)

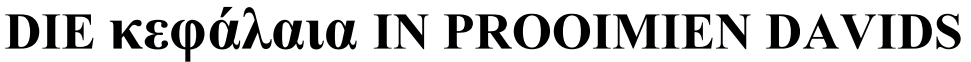 DES UNBESIEGBAREN: EIN VERGLEICH
}

Введения к неоплатоническим трактатам, посвящённым логике (Введение в философию, комментарии к «Введению» Порфирия, а также к «Об истолковании», «Категориям» и «Аналитике» Аристотеля), как правило, начинаются с рассмотрения общих характеристик

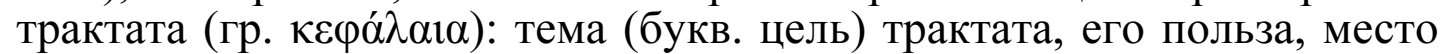
трактата в программе обучения логике, смысл названия, подлинность, отнесение трактата к определённому разделу философии, способ преподавания материала. В статье сопоставляются соответствующие отрывки введений к трактатам Давида Непобедимого «Комментарий к “Введению” Порфирия» и «Комментарий к “Аналитике” Аристотеля», а также армянские переводы обоих текстов.

Ключевые слова: неоплатонизм, Давид Непобедимый, переводы, древнегреческий, древнеармянский, античная философия, грекофильская школа, Аристотель, Аналитика.

E. V. Grigoryeva (Berlin, Germany)

\section{The prooemia of two treatises by David the Invincible: A Comparison}

Neoplatonic treatises on logics have a similar structure: they begin

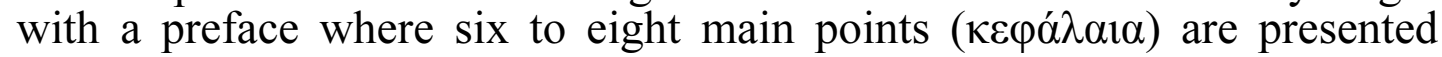
(aim of the treatise, its usefulness, reason for the title, division into chapters, the place of the treatise in the Aristotle's philosophy, its genuineness), then follows a textual commentary.

In the first section of this paper, the prooemia of two treatises by David the Invincible are compared (Commentary on Porphyry's Isagoge and Commentary on Aristotle's Analytics). In the second section, the Armenian translations of both texts are compared. It is shown that both translations have some similarities within the vocabulary and the usage of grammatical forms. However, the translation of the Commentary on the Analytics is more accurate than that of the Commentary on Isagoge. Whereas the translator of the Commentary on the Isagoge omits or changes certain phrases and words, the translator of the Commentary on the Analytics tries to maintain the translation as exact as possible.

However, there are more similarities between commentaries on the same treatise by different commentators (e.g. by David, Ammonius, and Philoponus) than between two commentaries on different treatises written by the same commentator.

Keywords: Neoplatonism, David the Invincible, Aristotle, Prior Analytics, commentary, translation, Old Armenian, Logics, Hellenizing School. 


\section{Einführung}

Schriften zur Logik wurden in den neuplatonischen Schulen in einer bestimmten Reihenfolge gelesen: nach einer allgemeinen Einführung in die Philosophie (Prolegomena philosophiae) folgte die Lektüre der Isagoge des Porphyrios, dann wurden drei Schriften des Aristoteles gelesen und kommentiert (De interpretatione, Kategorien, Analytik). Der Stil und der Inhalt der Texte variierten sich: Die ersten Schriften enthielten allgemeine Überlegungen über die Philosophie, Erklärungen einzelner Namen und Realien, die den Schülern eventuell nicht bekannt waren, sowie Zitate aus der griechischen Philosophie und Literatur. Das alles sollte das Eintauchen in die Philosophie einfacher machen. Die Kommentare zu den Schriften des Aristoteles waren dagegen wesentlich sachlicher und inhaltlich komplizierter.

Die Prooimien der neuplatonischen Schriften zur Logik (Prolegomena Philosophiae, Kommentar zur Isagoge des Porphyrios, Kommentare zu De interpretatione, zu den Kategorien und zu den Analytiken) haben normalerweise feste Struktur ${ }^{1}$. Am Anfang des

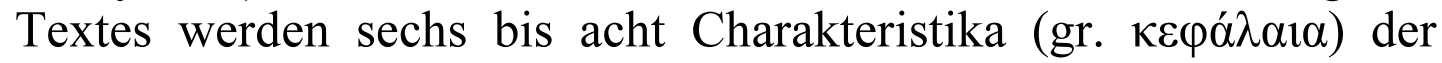

1 Über den neuplatonischen Kommentar s.: Hadot, Ilsetraut (Hg.): Simplicius. Sa vie, son oeuvre, sa survie. Actes du colloque international de Paris (28 sept.-ler oct. 1985), Berlin - New York 1987; Hadot, Ilsetraut: Les introductions aux commentaires exégétiques chez les auteurs néoplatoniciens et les auteurs chrétiens, in: M. Tardieu (Hg.): Les règles de l'interprétation, Paris 1987, 99-122; Hadot, Ilsetraut (Hg.): Simplicius, Commentaire sur les catégories. Trad. comm. sous la dir. de Ilsetraut Hadot, Philosophia antiqua 50, Leiden [u.a.]: Brill 1990. Bd.: 1. Introduction, P. 1-9,3 Kalbfleisch; Hadot, Ilsetraut: The Role of the Commentaries on Aristotle in the Teaching of Philosophy according to the Prefaces of the Neoplatonic Commentaries on the Categories, in: H. Blumenthal - H. Robinson (Hgg.), Aristotle and the later tradition (Oxford Studies in Ancient Philosophy, Supplementary Volume), Oxford 1991, 175-189; Hadot, Ilsetraut: Aristote dans l'enseignement philosophique néoplatonicien. Les préfaces des commentaires sur les Catégories, Revue de Théologie et de Philosophie 124 (1992), 407-425; Hadot, Ilsetraut: Le commentaire philosophique continu dans l'Antiquité, Antiquité Tardive 5 (1997), 169-176; Hoffmann, Philippe: What was Commentary in Late Antiquity? The Example of the Neoplatonic Commentators, in: M.-L. Gill - P. Pellegrin (Hgg.), The Cambridge Companion to Ancient Philosophy, Oxford 2007, 597-622; Sorabji, Richard: The ancient commentators on Aristotle in: R. Sorabji (Hg.): Aristotle Transformed. The Ancient Commentators and their Influence, Ithaca, NY 1990: 1-30. 
kommentierten Schrift aufgezählt ${ }^{2}$, die dann einzeln ausgelegt werden, und deren Zahl je nach dem Typ der zu kommentierenden Schrift variiert: das Ziel des Buches (ó $\sigma \kappa o \pi o ́ s)$, sein Nutzen ( $\tau$ ò

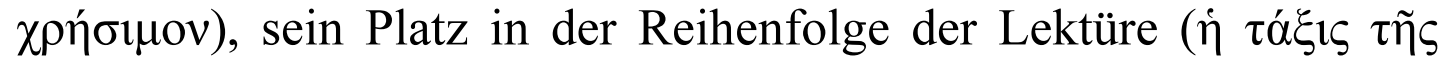

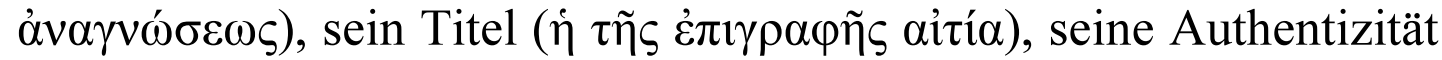

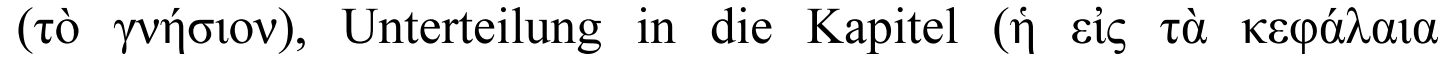

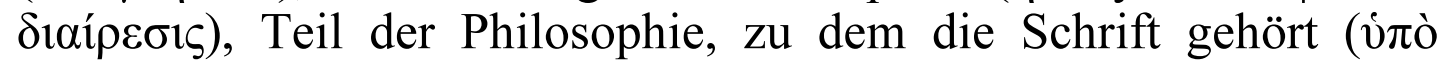

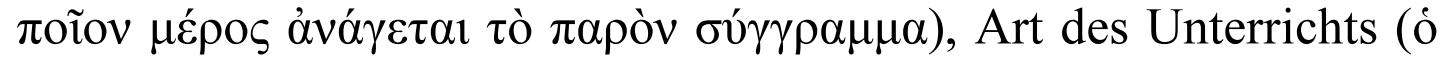
$\delta 1 \delta \alpha \sigma \kappa \alpha \lambda \imath \kappa o ̀ \varsigma \tau \rho o ́ \pi 0 \varsigma)$. Zum ersten Mal kann diese Struktur bei dem Neuplatoniker Ammonios festgestellt werden, wobei einzelne $\kappa \varepsilon \varphi \alpha ́ \lambda \alpha 1 \alpha$ sich auch früher finden lassen, z.B. in den Demosthenes-

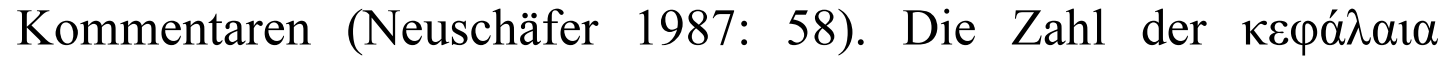
entwickelt sich im Laufe der Zeit von fünf zu acht: Im Kommentar des Ammonios zu De interpretatione (Ammon. in Int. 1,12-1,20) werden fünf $\kappa \varepsilon \varphi \alpha ́ \lambda \alpha 1 \alpha$ behandelt, in seinem Kommentar zu den Kategorien sind es sechs. Das siebte $\kappa \varepsilon \varphi \alpha ́ \lambda \alpha$ ov findet sich zum ersten Mal bei Ammonios im Kommentar zur Isagoge und dann bei Philoponos im Kommentar zur Analytik ${ }^{3}$, das achte bei David und Elias, jeweils im Kommentar zur Isagoge.

Für jede einzelne philosophische Schrift gab es seit einer gewissen Zeit eine festgelegte Zahl der $\kappa \varepsilon \varphi \alpha ́ \lambda \alpha 1 \alpha$ : in den Kommen-

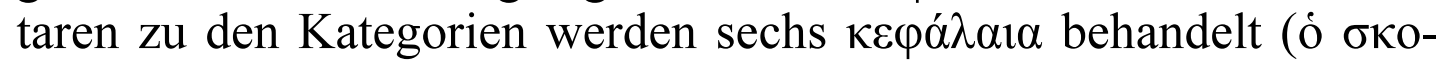

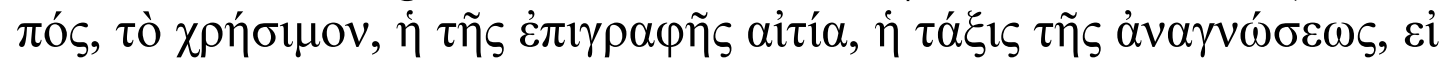

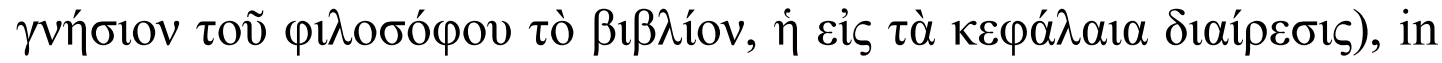
den Kommentaren zu den Analytiken wird auch über den Teil der

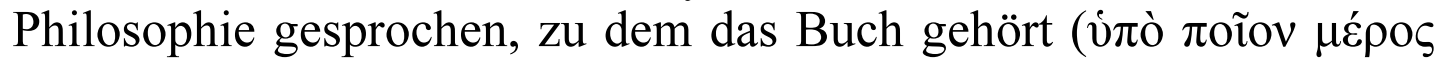

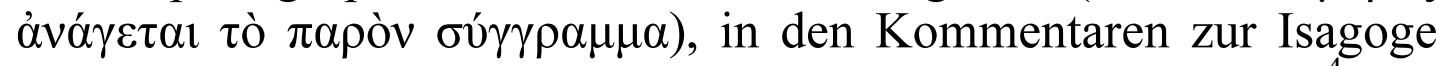

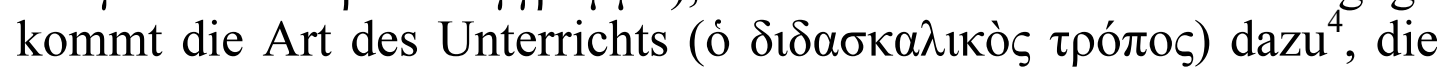
bei Ammonios noch fehlt (in Porph. 21,4-21,13) und zum ersten Mal bei David und Elias vorkommt ${ }^{5}$.

Im Folgenden werden die Abschnitte der Prooimien der Kommentare zur Isagoge und zur Analytik verglichen, in denen die $\kappa \varepsilon \varphi \alpha ́ \lambda \alpha 1 \alpha$ erörtert werden, mit dem Ziel, festzustellen, ob inhaltliche Gemeinsamkeiten in beiden Schriften Davids zu finden sind und ob

${ }^{2}$ Über die Prologe siehe: Neuschäfer 1987: 57-67 Anm. 1-57 (S. 355359); Hadot 1987a: 44-45.

${ }_{3}$ Ammon. in Porph. 21,4-21,13, Philop. in APr. 1,2-6.

${ }^{4}$ Über die unterschiedliche Zahl der $\kappa \varepsilon \varphi \alpha ́ \lambda \alpha \_$in den Kommentaren zu verschiedenen Schriften (acht in einer Einführung in die Philosophie, sechs in einem Kommentar zu den Kategorien bzw. zu der Analytik) s. Hadot 1990, 25-26.

${ }^{5}$ David, in Porph. 80,5-80,14, Elias in Porph. 35,1-35,13. 
eine besondere Ähnlichkeit beider Kommentare, über die schon Arevshatyan geschrieben hat (Arevshatyan 1967: 8), in diesen Textabschnitten festzustellen ist. Ferner werden die armenischen Übersetzungen beider Schriften analysiert und untereinander verglichen.

\section{Kommentar zur Isagoge}

Am Anfang des Prooimions werden acht $\kappa \varepsilon \varphi \alpha ́ \lambda \alpha 1 \alpha$ aufgelistet. In den Kapiteln zwei bis vier werden acht $\kappa \varepsilon \varphi \alpha ́ \lambda \alpha 1 \alpha$ behandelt: in einem relativ großen Kapitel zwei das Gesamtthema, im Kapitel drei der Nutzen, im Kapitel vier die Authentizität, der Platz in der Reihenfolge der Lektüre, die Unterteilung in die Kapitel, die Art des Unterrichts und ganz kurz der Teil der Philosophie, zu der das Werk gehört (Logik). Das Prooimion besteht ausschließlich aus der Darstellung der acht $\kappa \varepsilon \varphi \alpha ́ \lambda \alpha \iota \alpha$.

\section{Auslegung der Analytik}

Die sechs bzw. sieben $\kappa \varepsilon \varphi \alpha ́ \lambda \alpha 1 \alpha$ werden nicht, wie häufig in den Prologen $\mathrm{zu}$ den neuplatonischen Kommentaren, am Anfang aufgelistet, sie werden aber in den ersten zwei Kapiteln

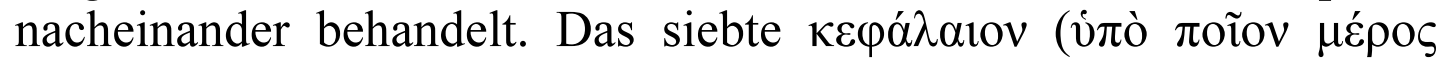

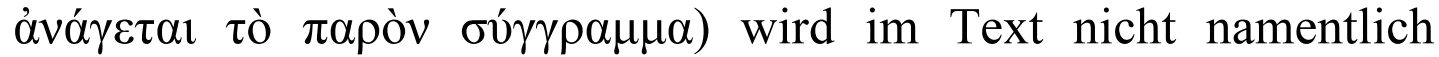
erwähnt, jedoch wird in den zwei darauffolgenden Kapiteln die Frage analysiert, ob die Logik ein Teil oder ein Instrument der Philosophie ist, was dem siebten $\kappa \varepsilon \varphi \alpha ́ \lambda \alpha$ เov inhaltlich nah ist.

Der Kommentar zur Analytik wurde im Unterschied zum Kommentar zur Isagoge nur auf Altarmenisch überliefert. Der altgriechische Text kann nur anhand der armenischen Übersetzung, anderer auf beiden Sprachen überlieferten Schriften Davids sowie zahlreicher neuplatonischer Kommentare zur Logik rekonstruiert

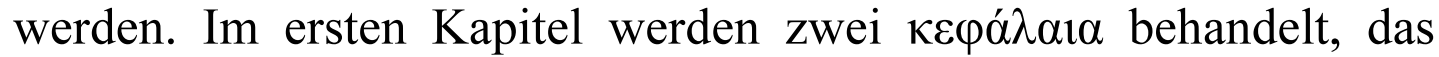
Gesamtthema und der Nutzen des Werkes. Im zweiten Kapitel

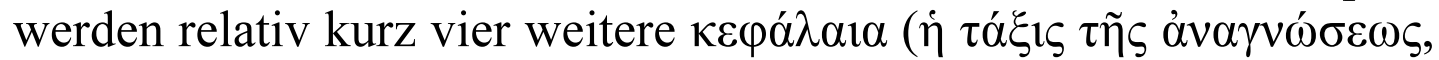

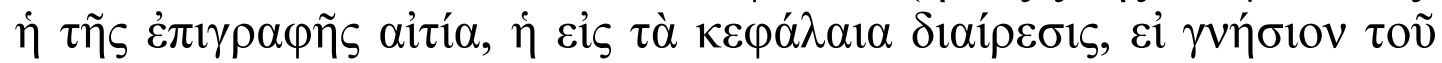

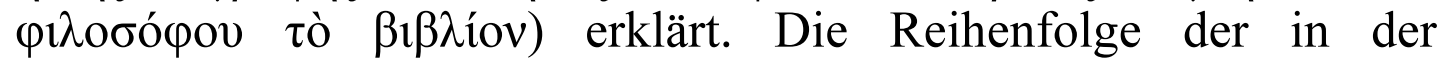
Einführung behandelten $\kappa \varepsilon \varphi \alpha ́ \lambda \alpha ı \alpha$ entspricht der im Kommentar des Philoponos. 


\section{Vergleich beider Textabschnitte}

Das Prooimion des Kommentars zur Analytik ist wesentlich kürzer als das Prooimion des Kommentars zur Isagoge und enthält, im Unterschied zum Kommentar zur Isagoge, keine Informationen über Aristoteles und seine Lehre sowie keine Zitate außer dem ersten Satz der Analytik (Topchyan 2010: 30,6-8). Man findet dagegen bestimmte Ähnlichkeiten zwischen dem Kommentar zur Analytik Davids einerseits und den Kommentaren zur Analytik des Ammonios und besonders des Philoponos andererseits: bei Philoponos ist der Abschnitt über die $\kappa \varepsilon \varphi \alpha ́ \lambda \alpha \iota \alpha$ zwar länger und ausführlicher als bei David, er hat aber ähnliche Struktur und Argumentation. So weisen zwei Kommentare zu derselben Schrift mehrere Ähnlichkeiten auf als zwei Kommentare eines Autors zu unterschiedlichen Werken. Die einzige auffallende Gemeinsamkeit beider Schriften David ist, dass das erste $\kappa \varepsilon \varphi \alpha ́ \lambda \alpha$ ovo, das Gesamtthema des Werkes, wesentlich ausführlicher dargestellt wird als alle anderen Charakteristika der Schrift.

\section{Armenische Übersetzungen beider Schriften}

Die Untersuchungen zur Vorgehensweise der armenischen Übersetzer haben gezeigt, dass die Genauigkeit der Übersetzung eines Werkes von der Autorität und der Stellung des Werkes im Philosophiestudium abhängig war. So wurden grundsätzlich die Werke der großen Philosophen (Platon, Aristoteles, Porphyrios) sehr genau, so wörtlich wie möglich übersetzt (Adontz 1915: CLXXXVI-CLXXXVII). Die neuplatonischen Texte, die einführenden Charakter hatten, wurden für den armenischen Leser adaptiert und in einigen Aspekten (griechische Realien, Namen, Reihen der Beispiele u.ä.) vereinfacht und teilweise gekürzt. Die Kommentare zu den Texten des Aristoteles (Kategorien, Analytik), die man nach diesen Einführungen las, wurden für die schon fortgeschrittenen Schüler wesentlich genauer wiedergegeben (ausführlich bei Calzolari 2007a, 2009b).

\section{Kommentar zur Isagoge 6}

Der Kommentar zur Isagoge wurde sowohl auf Altgriechisch als auch auf Armenisch überliefert, so dass man die Übersetzung mit dem griechischen Original vergleichen kann. Die Besonderheiten

\footnotetext{
${ }^{6}$ Alle Unterschiede zum griechischen Text des Abschnittes werden von Muradyan aufgelistet (Muradyan 2015: 65-75).
} 
der Übersetzung im Allgemeinen wurden bei Muradyan ausführlich dargestellt (Muradyan 2015: 26-51).

Im Folgenden werden die Kapitel zwei bis vier des Prooimions genau betrachtet, in denen acht $\kappa \varepsilon \varphi \alpha ́ \lambda \alpha 1 \alpha$ dargestellt werden. Insgesamt wurde der Text vom Übersetzer nicht wesentlich gekürzt (ungefähr $5 \%$ des griechischen Textes wurde vom Übersetzer ausgelassen, es geht vor allem um längere Erklärungen, die das schon Erklärte in einer anderen Form wiederholen). Einzelne Satzteile werden umgestellt, die grammatische Strukturen wie etwa Genitivi Absoluti bleiben dabei erhalten. Es gibt kaum Glossen oder andere Ergänzungen.

Das Verb $\delta 1 \alpha \lambda \alpha \mu \beta \alpha ́ v \omega$ bleibt mehrmals unübersetzt, teilweise wird es mit unterschiedlichen Verben (unqnuर, lupnuuuliuntud) wiedergegeben (siehe z.B. Muradyan 2015: 66, 10-14). Das Verb

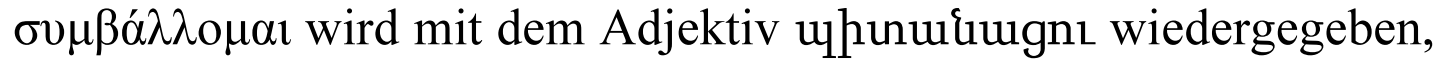

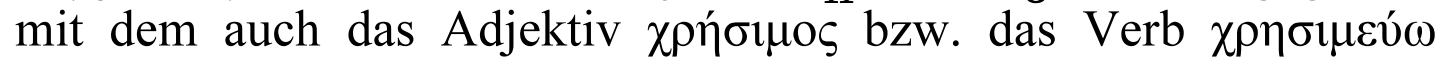
wiedergegeben wird.

Das griechische $\pi \rho \tilde{\omega} \tau o v$ wird an mehreren Stellen mit quupu $\mathrm{l}$ unuqhi wiedergegeben (z.B. Muradyan 2015: 76, 3, 5), genauso wie im Kommentar zur Analytik.

Tupàtuu, mit dem normalerweise $\pi \alpha ́ \lambda \imath v$ wiedergegeben wird, wird an einigen Stellen im Armenischen ergänzt, obwohl im Griechischen das Wort $\pi \alpha ́ \lambda \imath v$ fehlt (z.B. Muradyan 2015: 68, 11).

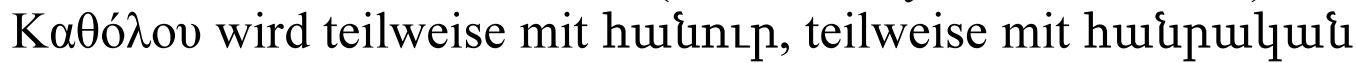
wiedergegeben. Im Kommentar zur Analytik dagegen wird ausschließlich huifuntp verwendet.

Die in griechischen Texten Davids sehr verbreiteten Kon-

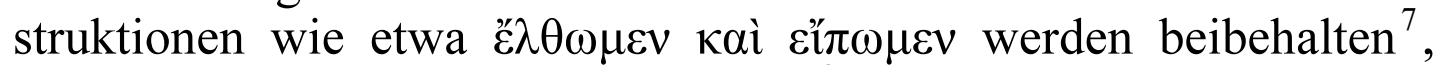
teilweise nur mit einem Verb übersetzt ${ }^{8}$, teilweise mit zwei, die aber im Armenischen weit voneinander stehen ${ }^{9}$.

An einigen Stellen werden die Personalpronomina und andere Wörter ergänzt ${ }^{10}$ bzw. an Stelle eines Personalpronomens ein Substantiv verwendet, was den Text verständlicher machen sollte. Griechische bestimmte Artikel werden an einigen Stellen mit Demonstrativpronomina übersetzt.

Besonders auffällig ist der Umgang des Übersetzers mit den Beispielen. Sie werden vom Übersetzer ersetzt, ergänzt oder

${ }_{8}^{7}$ Z.B. in Porph. 90,25 - vgl. Muradyan 2015: 84,6.

${ }^{8}$ Z.B. in Porph. 93,6 - vgl. Muradyan 2015: 88,8.

${ }^{9}$ Z.B. in Porph. 83,8-9 - vgl. Muradyan 2015: 64,33-34.

${ }^{10}$ Z.B. Uthq (Muradyan 2015: 74, 1,5; 77 Anm. e). 
gekürzt: Wenn an einer Stelle als Beispiel zwei griechische Namen vorkommen, bleibt nur ein in der Übersetzung ${ }^{11}$, die Lebewesen, die in den Beispielen vorkommen, werden durch andere ersetzt ${ }^{12}$.

Man kann im Allgemeinen nicht über eine Wort-für-WortÜbersetzung sprechen, der Genauigkeitsgrad ist jedoch sehr hoch.

\section{Kommentar zur Analytik}

Da das griechische Original verloren ist, kann man die Vorgehensweise des armenischen Übersetzers nur anhand der Parallelstellen aus anderen Texten Davids sowie aus anderen neuplatonischen Kommentaren rekonstruieren ${ }^{13}$. Man kann gewisse Vereinfachung des Textes feststellen: Verschiedene griechische Wörter werden mit einem armenischen übersetzt (z.B. alle Wörter mit der Bedeutung „sagen“, „fragen“, „antworten“" mit dem Wort uuku, ,reden"). Abschlussformel am Ende jedes Kapitels sind

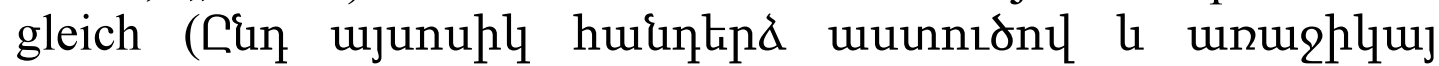
unpulpp), während man vermuten kann, dass im griechischen Text, jedes Kapitel mit unterschiedlichen Formeln beendet wird, die jeweils ,genug darüber“" bedeutet ${ }^{14}$. Allerdings ist diese Vereinfachung doch nicht so auffallend wie in den Übersetzungen der Prolegomena philosophiae und des Kommentars zur Isagoge: So bleiben z.B. die Namen, Realien und Beispiele, die wahrscheinlich im griechischen Original vorhanden waren, auch in der armenischen Übersetzung erhalten, was anhand der Parallelstellen in anderen neuplatonischen Kommentaren zur Logik (Ammonios, Simplikios, Philoponos, Elias) bewiesen werden kann.

Man kann ferner vermuten, dass die Verbalformen im Armenischen dem griechischen Original folgen ${ }^{15}$. Die Substantivierung

11 84, 19: Sokrates und Platon im griechischen Text, im armenischen dagegen nur Sokrates.

12 84, 3: im Griechischen werden Mensch und Pferd erwähnt, im Armenischen Adler und Hirsch (Muradyan 2015: 66,31).

${ }_{13}$ Ausführlicher s. Grigoryeva 2015.

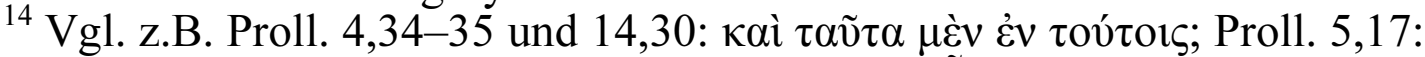

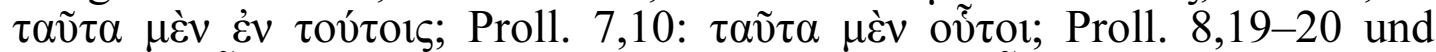

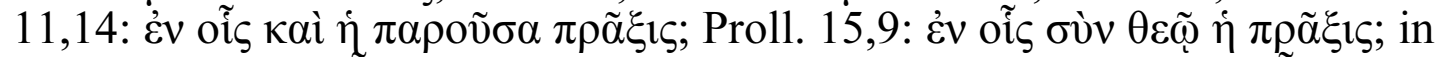

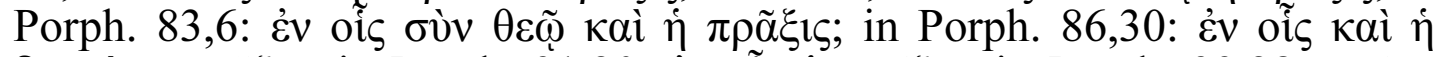
$\delta \varepsilon v \tau \varepsilon \dot{\varepsilon} \rho \pi \rho \tilde{\alpha} \xi 1 \zeta$; in Porph. 91,20: $\dot{\varepsilon} v$ oí $\tilde{\eta} \pi \rho \tilde{\alpha} \xi_{1} \zeta$; in Porph. 99,28: $\tau \alpha \tilde{v} \tau \alpha$

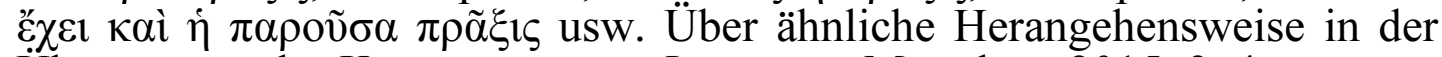
Übersetzung des Kommentars zur Isagoge s. Muradyan 2015: 3-4.

${ }^{15}$ 84,6-16: in dieser Passage werden substantivierte Infinitive (qn $\lfloor n j \mathrm{u} / \mathrm{n} / 2$ qn」nju) bzw. finite Formen (qki) verwenden, was auch dem Gebrauch von 
der Wörter und der Wortgruppen im Armenischen folgt wahrscheinlich dem griechischen Vorbild, was man auch aus vielen Parallelstellen schließen kann ${ }^{16}$.

An den Stellen, an denen die Form des griechischen Wortes nicht genau wiedergegeben werden kann, benutzt der Übersetzer passende armenische Wörter, wobei der Text ohne Vergleich mit entsprechenden Passagen in anderen Kommentaren zur Analytik nicht verstanden werden kann $^{17}$.

Es gibt einzelne Glossen des Übersetzers im Text, die einzelne Begriffe erklären und mit der Formel uju hipq (,das heißt“ vgl. gr. $\tau o v \tau \varepsilon ́ \sigma \tau \iota)$ eingeführt werden, etwa nach dem Model „Protasis d.h. Prämisse“.

Zusammenfassend lässt sich sagen, dass der armenische Übersetzer versucht hat, den griechischen Text der Ersten Analytik so wörtlich und genau wie möglich wiederzugeben. Die griechische Syntax und die griechische Grammatik werden im Allgemeinen nachgeahmt. In den Lemmata wird der Text so wörtlich wie möglich zitiert, an anderen Stellen im Kommentar gibt es dagegen Varianten.

\section{Vergleich beider Übersetzungen}

Bei der genaueren Betrachtung beider Übersetzungen konnten keine großen Unterschiede im Wortschatz (sowohl bei philosophischen Begriffen als auch bei allen anderen Wörtern ${ }^{18}$ ) entdeckt werden, es gibt auch keine Unterschiede in der Grammatik. Man kann vermuten, dass der Übersetzer des Kommentars zur Analytik

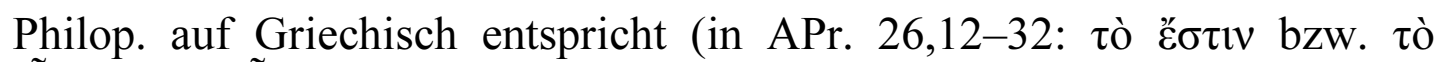

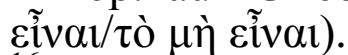

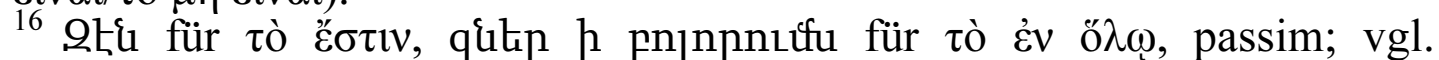
Topchyan 2010, 179-181; für in Porph. siehe Muradyan 2015, 40-42.

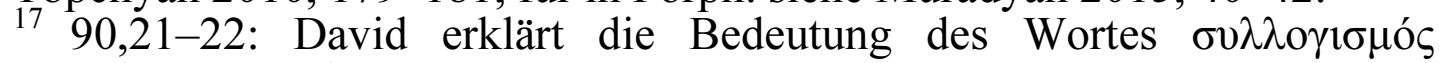
(punhuluupntưfu), indem er andere Wörter mit dem Präfix ovv- (arm. pun-) auflistet. Da es aber auf Armenisch keine Äquivalente zu diesen

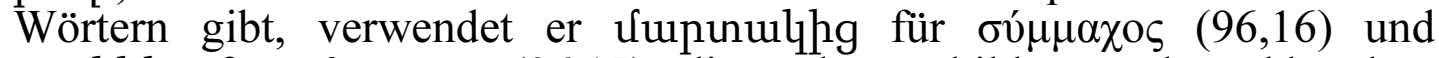
npntlihg für $\sigma 0 ́ v \tau \varepsilon \kappa v o \varsigma ~(96,17)$, die anders gebildet werden, d.h. ohne Präfixe, sondern mit dem Suffix -hg. Vgl. ähnliche Beispiele bei Alex. Aphr. in APr. 17,15-18; Ammon. in APr. 26,2-8.

${ }_{18}$ Eine Ausnahme ist das Wort '̌́oos, das im Kommentar zur Isagoge mit huluuuun, im Kommentar zur Analytik mit huiqutun wiedergegeben wird (z.B. Muradyan 2015: 78,36, vgl. Topchyan 2010, 100,3; 100,4; 100,5

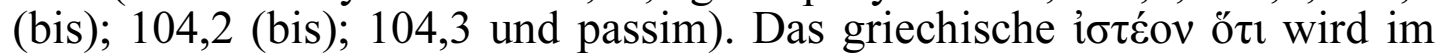
Kommentar zur Isagoge sowohl mit uqunu $t$ qhunt als auch mit qhun $\mathrm{t}$ übersetzt, im Kommentar zur Analytik ausschließlich mit uqunu $\mathrm{k}$ qhunt. 
dem Original treuer war als der Übersetzer des Kommentars zur Isagoge, mindestens auf dem Niveau der Beispiele sowie der griechischen Realien. Allerdings kann man nicht ausschließen, dass $\mathrm{zu}$ lange Erklärungen und Ergänzungen vom Übersetzer der Analytik genauso reduziert wurden wie vom Übersetzer des Kommentars zur Isagoge.

Es fällt auf, dass zwei Beispiele, die in beiden Kommentaren vorkommen, an der Stelle, an der der Begriff Induktion erklärt wird, auf Armenisch identisch aussehen bzw. nur sehr kleine Unterschiede haben. Im ersten Beispiel werden die Tiere aufgezählt, die den Unter- bzw. den Oberkiefer bewegen. Im griechischen Kommentar zur Isagoge werden Phoenix, Krokodil und Delphin erwähnt ${ }^{19}$, der Übersetzer lässt den Delphin aus. Im Kommentar zur Analytik dagegen werden Phoenix, Krokodil und Delphin erwähnt ${ }^{20}$. Im zweiten Beispiel geht es um Peisistratos, Dionysios und die Leibwächter. Im armenischen Text sind beide Beispiele praktisch identisch $^{21}$. Zwei Beispiele werden in unterschiedlicher Reihenfolge angegeben: im Kommentar zur Isagoge kommt zuerst das Beispiel mit den Tieren, im Kommentar zur Analytik das Beispiel mit Peisistratos und Dionysios.

Auch wenn es einige Gemeinsamkeiten in beiden Schriften auf Griechisch und auf Armenisch nicht zu übersehen sind, kann man anhand dieser Ähnlichkeiten keine Aussagen bzw. zusätzliche Argumente über die Autorschaft beider Schriften, d.h. über ihre Zugehörigkeit einem Autor machen. Man kann auch keine Aussagen darüber machen, ob beide Texte von einem oder von zwei verschiedenen Übersetzern ins Armenische übertragen wurden.

\section{Primärliteratur:}

\section{Literatur}

David 1904: Davidis Prolegomena et in Porphyrii Isagogen commentarium, edidit Adolfus Busse, Commentaria in Aristotelem Graeca, 18.2. Berlin: Reimer.

Arevshatyan, S. S. 1967: [David the Invincible, Commentary on Aristotle's Analytics], Yerevan: Izdatelstvo AN Armyanskoy SSR. Tuuhp

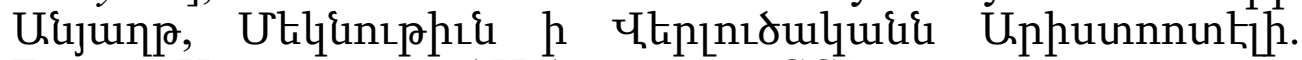
Ереван: Издательство АН Армянской ССР.

\footnotetext{
${ }^{19}$ In Porph. 89,5-6, Muradyan 2015: 80,15.

20 Topchyan 2010: 100, 11. Über Delphin s. Topchyan 2010: 101 Anm. 207.

${ }^{21}$ Topchyan 2010: 100,5-7; 100,7-8; 100,9-10, Muradyan 2015: 78,1-3; 80,23-24; 78,34-35.
} 
Topchyan, A. 2010: David the Invincible, Commentary on Aristotle's Prior Analytics, Old Armenian Text with an English Translation, Introduction and Notes by Aram Topchyan. Philosophia antiqua 122, Commentaria in Aristotelem Armeniaca. Davidis Opera 2. Leiden: Brill.

Muradyan, G. 2015: David the Invincible, Commentary on Porphyry's Isagoge. Old Armenian Text with the Greek Original, an English Translation, Introduction and notes by Gohar Muradyan. Philosophia antiqua 137, Commentaria in Aristotelem Armeniaca. Davidis Opera 3. Leiden: Bril.

\section{Sekundärliteratur:}

Adontz, N. 1915: [Adontz, Nikolaj: Dionisij Frakijskij i armianskie tolkovateli]

Адонц, Н. 1915: Дионисий Фракийский и армянские толкователи, Ars Dionysii Grammatici et Armeniaca in eam scholia Davidis Philosophi, rec. et disputavit N. Adontz, Bibliotheca Armeno-Georgica 4. СПб.: Типография Академии наук.

Calzolari, V. (Hg.) 2007a: Illuminations d'Arménie. Arts du livre et de la pierre dans l'Arménie ancienne et médiévale (Livre-catalogue de l'exposition 'Illuminations d'Arménie', Fondation Martin Bodmer, Cologny-Genève, 15 septembre-30 décembre 2007). Genève 2007.

Calzolari, V. 2007b: Un philosophe 'Invincible': le néoplatonicien David, in: Calzolari 2007a, 195-199.

Calzolari, V., Barnes, J. (Hg.) 2009a: L'oeuvre de David l'Invincible et la transmission de la pensée grecque dans la tradition arménienne et syriaque. Boston; Köln: Brill.

Calzolari, V. 2009b: David et la tradition arménienne, in: Calzolari 2009a, 20-48.

Grigoryeva, E. 2015: Der armenische Text des Aristoteles in der "Auslegung der ,Analytiken"" Davids. Indoevropeiskoe yazykoznanie i klassicheskaya filologiya [Indo-European linguistics and classical philology]. 19, 179-192.

Hoffmann, Ph. 2007: What was Commentary in Late Antiquity? The Example of the Neoplatonic Commentators, in: M.-L. Gill, P. Pellegrin (Hgg.). The Cambridge Companion to Ancient Philosophy. Oxford, 597-622.

Mahé, J.-P. 1990: David l'Invincible dans la tradition arménienne. In: Simplicius: Commentaire sur les Catégories, traduction commentée sous la direction de Ilsetraut Hadot Bd. 1. Leiden; New York; København; Köln: Brill, 189-207.

Muradyan, G. 2009a: David the Invincible's Commentary on Porphyry's Isagoge. A Collation of the Greek and Armenian Versions. In: Calzolari 2009a, 91-117.

Muradyan, G. 2012: Grecisms in Ancient Armenian. Hebrew University Armenian Studies 13. Leuven: Peeters.

Muradyan, G. 2014: The Hellenizing School. In: Armenian Philology in the Modern Era. From Manuscript to Digital Text. Ed. by V. Calzolari with the Collaboration of M. E. Stone. Leiden: Brill, 321-348. 
Neuschäfer, B. 1987: Origenes als Philologe. Schweizerische Beiträge zur Altertumswissenschaft 18, Bdd. 1-2. Basel: Reinhardt.

Sgarbi, R. 1972: Osservazioni sul testo e sulla lingua della versione armena dell' 'Isagoge' di Porfirio. Memorie dell'Istituto Lombardo. Accademia di Scienze e Lettere. Classe di Lettere, Scienze morali e storiche, vol. 31, fasc. 5. Milano.

Sorabji, R. (Hg.) 1990: Aristotle Transformed. The Ancient Commentators and Their Influence. Ithaca, NY.

Westerink, L. G. 1990: The Alexandrian Commentators and the Introductions to Their Commentaries. In: Sorabji 1990, 325-348.

Wildberg, C. 1990: Three Neoplatonic Introductions to Philosophy: Ammonios, David, Elias. Hermathena 149, 33-51. 